\title{
Transient obscuration event captured in NGC 3227
}

\section{Continuum model for the broadband spectral energy distribution}

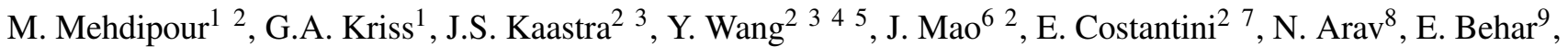 \\ S. Bianchi ${ }^{10}$, G. Branduardi-Raymont ${ }^{11}$, M. Brotherton ${ }^{12}$, M. Cappi ${ }^{13}$, B. De Marco ${ }^{14}$, L. Di Gesu ${ }^{15}$, J. Ebrero ${ }^{16}$, \\ S. Grafton-Waters ${ }^{11}$, S. Kaspi ${ }^{17}$, G. Matt ${ }^{10}$, S. Paltani ${ }^{18}$, P.-O. Petrucci ${ }^{19}$, C. Pinto ${ }^{20}$, G. Ponti ${ }^{21}{ }^{22}$, F. Ursini ${ }^{13}$, and \\ D.J. Walton ${ }^{23}$
}

1 Space Telescope Science Institute, 3700 San Martin Drive, Baltimore, MD 21218, USA e-mail: mmehdipour@stsci.edu

2 SRON Netherlands Institute for Space Research, Sorbonnelaan 2, 3584 CA Utrecht, the Netherlands

3 Leiden Observatory, Leiden University, PO Box 9513, 2300 RA Leiden, the Netherlands

${ }^{4}$ CAS Key Laboratory for Research in Galaxies and Cosmology, Department of Astronomy, University of Science and Technology of China, Hefei 230026, China

5 School of Astronomy and Space Science, University of Science and Technology of China, Hefei 230026, China

6 Department of Physics, University of Strathclyde, Glasgow G4 0NG, UK

7 Anton Pannekoek Institute, University of Amsterdam, Postbus 94249, 1090 GE Amsterdam, The Netherlands

8 Department of Physics, Virginia Tech, Blacksburg, VA 24061, USA

9 Department of Physics, Technion-Israel Institute of Technology, 32000 Haifa, Israel

10 Dipartimento di Matematica e Fisica, Università degli Studi Roma Tre, via della Vasca Navale 84, 00146 Roma, Italy

11 Mullard Space Science Laboratory, University College London, Holmbury St. Mary, Dorking, Surrey, RH5 6NT, UK

12 Department of Physics and Astronomy, University of Wyoming, Laramie, WY 82071, USA

13 INAF-IASF Bologna, Via Gobetti 101, I-40129 Bologna, Italy

14 Departament de Física, EEBE, Universitat Politècnica de Catalunya, Av. Eduard Maristany 16, E-08019 Barcelona, Spain

15 Italian Space Agency (ASI), Via del Politecnico snc, 00133, Roma, Italy

16 Telespazio Vega UK for the European Space Agency (ESA), European Space Astronomy Centre (ESAC), Camino Bajo del Castillo,s/n, E-28692 Villanueva de la Cañada, Madrid, Spain

17 School of Physics and Astronomy and Wise Observatory, Tel Aviv University, Tel Aviv 69978, Israel

18 Department of Astronomy, University of Geneva, 16 Ch. d Ecogia, 1290 Versoix, Switzerland

19 Univ. Grenoble Alpes, CNRS, IPAG, 38000 Grenoble, France

20 INAF-IASF Palermo, Via U. La Malfa 153, I-90146 Palermo, Italy

21 INAF-Osservatorio Astronomico di Brera, Via E. Bianchi 46, I-23807 Merate (LC), Italy

22 Max Planck Institute fur Extraterrestriche Physik, 85748, Garching, Germany

23 Institute of Astronomy, University of Cambridge, Madingley Road, Cambridge CB3 OHA, UK

Received 17 May 2021 / Accepted 24 June 2021

\begin{abstract}
From Swift monitoring of a sample of active galactic nuclei (AGN) we found a transient X-ray obscuration event in Seyfert-1 galaxy NGC 3227, and thus triggered our joint XMM-Newton, NuSTAR, and Hubble Space Telescope (HST) observations to study this event. Here in the rst paper of our series we present the broadband continuum modelling of the spectral energy distribution (SED) for NGC 3227, extending from near infrared (NIR) to hard X-rays. We use our new spectra taken with XMM-Newton, NuSTAR, and the HST Cosmic Origins Spectrograph (COS) in 2019, together with archival unobscured XMM-Newton, NuSTAR, and HST Space Telescope Imaging Spectrograph (STIS) data, in order to disentangle various spectral components of NGC 3227 and recover the underlying continuum. We nd the observed NIR-optical-UV continuum is explained well by an accretion disk blackbody component $\left(T_{\max }=10 \mathrm{eV}\right)$, which is internally reddened by $E\left(\begin{array}{ll}B & V\end{array}\right)=045$ with a Small Magellanic Cloud (SMC) extinction law. We derive the inner radius $\left(12 R_{\mathrm{g}}\right)$ and the accretion rate $\left(0.1 \mathrm{M} \mathrm{yr}^{1}\right)$ of the disk by modelling the thermal disk emission. The internal reddening in NGC 3227 is most likely associated with out ows from the dusty AGN torus. In addition, an unreddened continuum component is also evident, which likely arises from scattered radiation, associated with the extended narrow-line region (NLR) of NGC 3227. The extreme ultraviolet (EUV) continuum, and the soft X-ray excess, can be explained with a warm Comptonisation component. The hard X-rays are consistent with a power-law and a neutral re ection component. The intrinsic bolometric luminosity of the AGN in NGC 3227 is about $2210^{43} \mathrm{erg} \mathrm{s}^{1}$ in 2019, corresponding to $3 \%$ Eddington luminosity. Our continuum modelling of the new triggered data of NGC 3227 requires the presence of a new obscuring gas with column density $N_{\mathrm{H}}=5 \quad 10^{22} \mathrm{~cm}^{2}$, partially covering the X-ray source $\left(C_{f}=06\right)$.
\end{abstract}

Key words. X-rays: galaxies - galaxies: active - galaxies: Seyfert - galaxies: individual: NGC 3227 - accretion disks - techniques: spectroscopic 


\section{Introduction}

Long-term monitoring of X-ray spectral variability in type-1 AGN with the Rossi X-ray Timing Explorer (RXTE) and Swift observatories have revealed that sometimes they display sudden spectral hardening events (see e.g. Markowitz et al. 2014; Kaastra et al. 2014; Mehdipour et al. 2017). These events are seen as spikes in the X-ray hardness ratio lightcurves, which typically last for days or weeks, or even several years in the case of NGC 5548. Over the past few years new results regarding the nature and origin of these transient spectral hardening events in type-1 AGN suggest they are caused by obscuring winds from the accretion disk, as rst discovered in NGC 5548 (Kaastra et al. 2014). Simultaneous multi-wavelength spectroscopy in the UV and X-ray energy bands has been instrumental for the study of these events. Transient X-ray obscuration in AGN is found to appear with an associated UV broad absorption-line component (Kriss et al. 2019), and in some cases with a high-ionisation component in the Fe K-band (Mehdipour et al. 2017).

In 2015 we started a monitoring program with the Neil Gehrels Swift Observatory (Gehrels et al. 2004) to observe the X-ray hardness variability in a sample of Seyfert-1 AGN, in order to trigger joint ToO observations with XMM-Newton (Jansen et al. 2001), NuSTAR (Harrison et al. 2013), and the Hubble Space Telescope (HST) Cosmic Origins Spectrograph (COS, Green et al. 2012). In December 2016 we found an event in NGC 3783 and triggered our observations. Mehdipour et al. (2017) show that the spectral hardening is caused by an obscuring wind, out owing with a velocity of few thousand $\mathrm{km} \mathrm{s}^{1}$, that crosses our line of sight. These obscuring winds are remarkably di erent from the commonly-seen warm-absorber out ows in AGN (e.g. Blustin et al. 2005; Laha et al. 2014). They have higher column densities and out ow velocities, and are located in the broad-line region (BLR) rather the narrow-line region (NLR). The optically-thick obscuration shields much of the Xray radiation, which has important impacts on the ionisation state and our interpretation of both the warm absorber (Arav et al. 2015) and the BLR (Dehghanian et al. 2019).

Following our ndings in NGC 3783, we continued our Swift monitoring to nd more of these events in other AGN to broaden our understanding of this transient obscuration phenomenon. In 2019 we discovered another obscuration event in the AGN NGC 3227. Figure 1 shows the Swift light curve of NGC 3227 from March 2018 to end of 2019. In November 2019, we found an intense X-ray spectral hardening event that lasted for a few weeks until the end of the Swift visibility window. During this period we successfully executed the triggering of our XMMNewton, NuSTAR, and HST/COS observations. Two sets of observations were taken, separated by about three weeks. Figure 2 compares an archival unobscured spectrum of NGC 3227 taken on 5 December 2016 with the new obscured spectra taken on 15 November and 5 December 2019. Strong soft X-ray absorption is evident in the new spectra. There is also intrinsic X-ray variability between the two 2019 observations.

NGC 3227 is a Seyfert-1 galaxy at redshift of 0.003859 (de Vaucouleurs et al. 1991) based on H i 21-cm line measurements. From an XMM-Newton and NUSTAR campaign carried out in 2016, Turner et al. (2018) found occultation by gas clouds that cause rapid X-ray ux and spectral variability. They concluded that the occulting gas is likely associated with clouds in the inner BLR. The X-ray variability and lags seen during the campaign were further studied by Lobban et al. (2020). The spiral galaxy of NGC 3227 is interacting with its companion galaxy NGC 3226 , which is a dwarf elliptical galaxy. The $\mathrm{H}$ i radio mapping studies

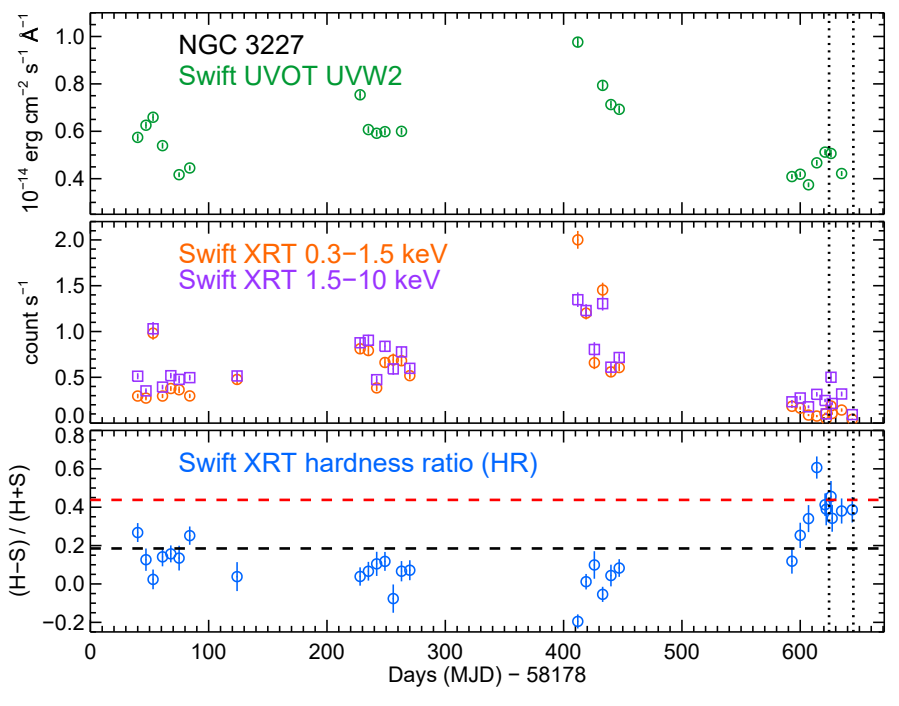

Fig. 1. Swift light curve of NGC 3227 from March 2018 to end of 2019. The dashed black line in the bottom panel indicates the average quiescent hardness ratio (HR) from unobscured data. The dashed line in red is the HR limit for triggering, above which signi cant obscuration was predicted according to our simulations. The hardness ratio is de ned as $\left(\begin{array}{ll}H & S\end{array}\right)(H+S)$, where $H$ and $S$ are the $S$ wift XRT count rate uxes in the hard (1.5-10 keV) and soft (0.3-1.5 keV) bands, respectively. The rst and second XMM-Newton observations, taken on 15 November and 5 December 2019, are indicated by vertical dotted lines.

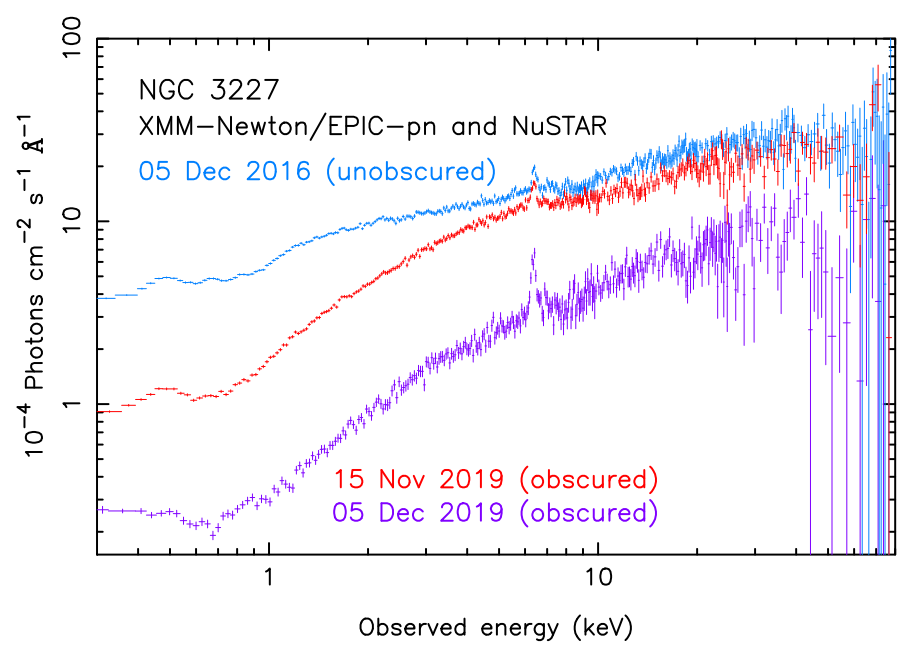

Fig. 2. NGC 3227 unobscured (2016) and obscured (2019) spectra from XMM-Newton EPIC-pn and NuSTAR. The displayed energy for EPICpn is $0.3-10 \mathrm{keV}$ and for NUSTAR $10-80 \mathrm{keV}$.

of NGC 3227 show that there is a signi cant column density of neutral gas in the host galaxy of NGC 3227 as a result of tidal interactions between the two galaxies (Mundell et al. 1995b). Signi cant internal reddening is also evident from the opticalUV spectrum of NGC 3227 (Crenshaw et al. 2001).

In this paper (Paper I) we determine the intrinsic broadband continuum of the spectral energy distribution (SED) in NGC 3227, while in our following papers we study the ionised out ows (Paper II by Wang et al. 2021), investigate the new obscuring wind in detail (Paper III by Mao et al. in prep), and analyse the nature of variability in NGC 3227 (Paper IV by Grafton-Waters et al. in prep). Establishing a broadband continuum model is important for (1) correctly disentangling the spec- 
tral components of obscuration from the continuum; (2) photoionisation modelling of the ionised AGN out ows, and correctly deriving and interpreting the AGN wind parameters; (3) understanding how accretion leads to the production of the observed broadband continuum emission. In order to derive the continuum, all foreground e ects in our line of sight to the central engine of the AGN, such as reddening and absorption, which are prominent in NGC 3227, need to be properly taken into account. This means broadband continuum modelling and absorption/reddening modelling are dependent on each other.

The structure of the paper is as follows. The observations and the reduction of data are described in Sect. 2. The spectral analysis and modelling of the continuum, reddening, and absorption are presented in Sect. 3. We discuss our results about the origin of the internal reddening and the components of the accretionpowered SED in Sect. 4, and give concluding remarks in Sect. 5 . We adopt a luminosity distance of $16.58 \mathrm{Mpc}$ in our calculations with the cosmological parameters $H_{0}=70 \mathrm{~km} \mathrm{~s}{ }^{1} \mathrm{Mpc}{ }^{1}$,

$=070$, and ${ }_{m}=030$. We assume proto-solar abundances of Lodders et al. (2009) in all our computations in this paper.

\section{Observations and data processing}

The observation log of data used in our SED modelling are provided in Table 1. We model two sets of spectra: the unobscured spectra taken by XMM-Newton and NuSTAR on 5 December 2016, and the obscured spectra taken by XMM-Newton and $\mathrm{NuS}$ $T A R$ on 5 December 2019. Hereafter we refer to them as the unobscured 2016 and the obscured 2019 observations. The 2016 unobscured spectra help us in better constraining the model for the intrinsic soft X-ray continuum, which is strongly absorbed in 2019. To determine the intrinsic broadband continuum, we also use the simultaneous HST/COS UV spectrum taken on 5 December 2019, as well as an archival HST Space Telescope Imaging Spectrograph (STIS) spectrum taken in 2000 in order to extend the spectral coverage to optical and near infrared (NIR) energies. As described in Sect. 1 two sets of observations were taken in 2019 (Fig. 2). We use the second set (5 December 2019), which is su cient for our purpose of obtaining the SED at the obscured epoch. Also, the second obscured spectrum is the one that di ers the most from the unobscured 2016 spectrum (Fig. 2), thus can be more useful for checking di erences in the SEDs of two epochs. Furthermore, the XMM-Newton Optical Monitor (OM) grism spectrum of the rst 2019 observation (15 November 2019) had contamination from another source, and thus we use the second 2019 observation (5 December 2019). We describe the processing and preparation of data from di erent observatories in the following.

\subsection{NIR-optical-UV data}

Our new HST observations of NGC 3227 used COS (Green et al. 2012) to obtain UV spectra covering the far-UV spectral range from 1067 to $1801 \AA$. We obtained four $415 \mathrm{~s}$ exposures at all four focal-plane positions (FP-POS) with grating G130M at a central wavelength setting of $1222 \AA$; two 480 s exposures with grating G160M with central wavelength $1533 \AA$; and two $564 \mathrm{~s}$ exposures with grating G160M with central wavelength $1623 \AA$. The diverse grating settings and focal plane positions enable us to span the gaps between detector segments A and B as well as eliminate at- eld artifacts and other detector anomalies (Dashtamirova \& Fischer 2020). We retrieved the COS data from the
Mikulski Archive for Space Telescopes (MAST) as processed with the latest calibration pipeline, v3.3.10.

In order to fully determine the shape of the NIR-opticalUV continuum, we also retrieved archival spectra using the HST Space Telescope Imaging Spectrograph (STIS) (Woodgate et al. 1998), which extends the spectral coverage to $10255 \AA$. The STIS spectrum was scaled by a factor of 0.68 to match the 2019 COS ux at their overlapping energy bands. These data were also processed with the standard data reduction pipelines. The observation log of the HST COS and STIS observations, and the XMM-Newton and NUSTAR observations that were used in our SED modelling, are provided in Table 1.

In our SED modelling we made use of XMM-Newton OM (Mason et al. 2001) data. The data from OM, which was operated in the Science User De ned mode, were taken with the primary photometric lters and the optical grism. The size of the circular aperture used for our photometry was set to a diameter of 12 , which is the optimum aperture size based on the calibration of these instruments. For a description of the reduction of OM data, we refer to Appendix A in Mehdipour et al. (2015) and references therein, which also applies to the data used here.

In this paper we also made use of the NIR continuum uxes that were derived by Kishimoto et al. (2007) from archival HST Near Infrared Camera and Multi-Object Spectrometer (NICMOS) observations, taken with the F160W (16050 $\AA$ ) and F222M (22180 ̊) lters. We use these two continuum data points to compare with our SED model in the NIR band as described in Sect. 3.1.

\subsection{X-ray data}

For our SED modelling in the X-ray band, we tted spectra from XMM-Newton RGS and EPIC-pn, and NuSTAR. The XMMNewton data were processed using the Science Analysis System (SAS v18.0.0). The RGS (den Herder et al. 2001) instruments were operated in the standard Spectro+Q mode. The data were processed through the rgsproc pipeline task; the source and background spectra were extracted and the response matrices were generated. We ltered out time intervals with background count rates $>01$ count $^{1}{ }^{1}$ in CCD number 9. The rst-order RGS1 and RGS2 spectra were tted simultaneously over the 6$37 \AA$ band in our spectral modelling.

The XMM-Newton EPIC-pn instrument (Strüder et al. 2001) was operated in the Small-Window mode with the Thin Filter. Periods of high- aring background for EPIC-pn (exceeding 0.4 count $\mathrm{s}^{1}$ ) were ltered out while applying the \#XMMEA_EP lter. We extracted a single event (PATTERN==0), high-energy ( $P I>10000$ \&\& $P I<12000$ ) light curve from the event le over the entire chip to identify intervals of high- aring background. The XMM-Newton EPIC-pn spectra were extracted from a circular region centred on the source with a radius of 40 . The background was extracted from a nearby source-free region of radius 40 on the same CCD as the source. The pileup was evaluated to be negligible. The single and double events were selected for the EPIC-pn (PATTERN <= 4). Instrumental response matrices were generated for the spectrum using the rmfgen and arfgen tasks. The tted spectral range for EPIC-pn is $0.3-10 \mathrm{keV}$.

The NUSTAR observations were reduced using the NuSTAR Data Analysis Software (NUSTARDAS) and CALDB calibration les of HEASoft v6.27. The data were processed with the standard pipeline script nupipeline to produce level 1 calibrated and level 2 cleaned event les. The data from the South Atlantic Anomaly passages have been ltered out and event les 
Table 1. Log of the NGC 3227 observations used in this paper for our SED modelling.

\begin{tabular}{l|ccc}
\hline \hline Observatory & Obs. ID & $\begin{array}{c}\text { Obs. date } \\
\text { yyy-mm-dd }\end{array}$ & $\begin{array}{c}\text { Length } \\
(\mathrm{ks})\end{array}$ \\
\hline HST COS & 15673 & $2019-12-05$ & 3.7 \\
HST STIS & 8479 & $2000-02-08$ & 4.0 \\
\hline XMM-Newton & 0782520601 & $2016-12-05$ & 85 \\
XMM-Newton & 0844341401 & $2019-12-05$ & 51 \\
\hline NuSTAR & 60202002010 & $2016-12-05$ & 41 \\
NuSTAR & 80502609004 & $2019-12-05$ & 28 \\
\hline
\end{tabular}

Notes. The dates correspond to the start time of the observations. The HST COS observation was taken with the G130M and G160M gratings, and the STIS observation with the G140L, G230L, G430L, and G750L gratings.

were cleaned with the standard depth correction, which reduces the internal background at high energies. The source was extracted from a circular region (radius $\sim 90^{\prime \prime}$ ), with the background extracted from a source-free area of equal size on the same detector. Then the nuproducts script was run to create level 3 products (spectra, lightcurves, ARF and RMF response files) for each of the two hard X-ray telescope modules (FPMA and FPMB) onboard NUSTAR. The spectra and corresponding response files of the two telescopes were combined for spectral modelling using the mathpha, addrmf, and addarf tools of the HEASOFT package. The fitted spectral range for NUSTAR is 5-78 $\mathrm{keV}$.

Our spectral analysis and modelling were done using the SPEX package (Kaastra et al. 1996; Kaastra et al. 2020) v3.06 with C-statistics for fitting the X-ray spectra and $\chi^{2}$ fitting for the NIR-optical-UV data. The model parameter errors are given at the $1 \sigma$ confidence level. The XMM-Newton and NUSTAR spectra are optimally binned according to Kaastra \& Bleeker (2016) for fitting in SPEX.

\section{Spectral analysis and modelling of the spectral energy distribution}

Here we present our modelling of the spectral components that form the observed SED in NGC 3227. We jointly model the spectra of the 2016 (unobscured) and 2019 (obscured) observations. We derive the intrinsic NIR-optical-UV-X-ray continuum by modelling all the reddening and X-ray absorption in our line of sight towards the nucleus of NGC 3227. Our spectral modelling is not affected by the companion galaxy NGC 3226 as it is far enough from NGC 3227.

\subsection{NIR-optical-UV continuum and reddening}

The NIR-optical-UV spectrum of NGC 3227 is shown in Fig. 3 . To fit the continuum emission extending from NIR to UV we started with a disk blackbody model (dbb in SPEX). This model is based on a geometrically thin, optically thick, ShakuraSunyaev accretion disk model (Shakura \& Sunyaev 1973). This model is appropriate for the accretion disk of the Seyfert-1 galaxy NGC 3227 and fits well the continuum of HST COS and STIS spectra over 1067-10255 $\AA$, after including in the model the effect of reddening described below.

The 2016 unobscured observation does not have any HST data and its XMM-Newton OM exposure was taken only with

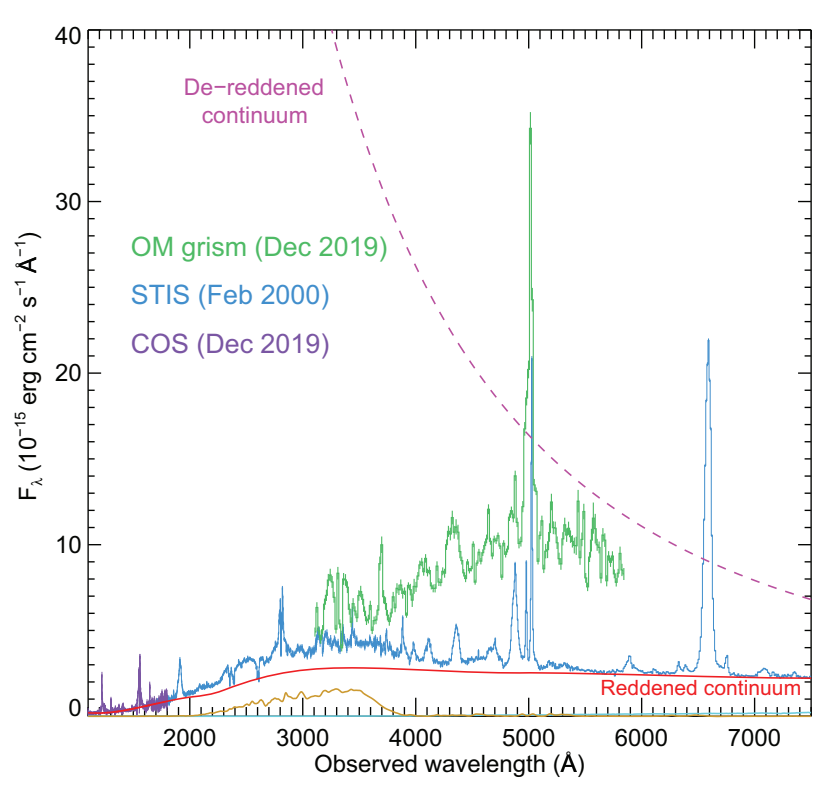

Fig. 3. Overview of the reddened NIR-optical-UV spectrum of NGC 3227, taken with HST COS and STIS, and XMM-Newton OM. The best-fit reddened disk blackbody model $(\mathrm{dbb})$ is shown in solid red line. For comparison the dbb model without the effect of reddening is shown in dashed magenta line. The model component with a broad feature shown in solid dark yellow line is the blended Fe II and Balmer emission. The strong excess emission in the OM optical grism spectrum relative to the STIS spectrum is the host galaxy emission captured by the larger OM aperture. The host galaxy emission taken by the HST aperture is negligible, shown by the model component in cyan.

the UVW1 filter. Therefore, in our SED modelling we assume the shape of the NIR-optical-UV continuum in the 2016 observation was the same as in the 2019 one, and simply scale the normalisation of the dbb model (derived from the 2019 observation) to match the 2016 OM flux. The UVW1 flux in the 2016 observation was higher by a factor of 1.82 than in the 2019 observation, thus we use this flux ratio for scaling the $\mathrm{dbb}$ model.

NGC 3227 displays significant internal reddening. This is evident from both the shape of the observed optical-UV continuum and the flux ratio of the AGN emission lines. As shown in the optical-UV spectrum of Fig. 3, and later in the SED plot of Fig. 7 (top panel), the observed optical-UV continuum drops steeply towards higher energies. This is characteristic of strong reddening. The Milky Way reddening in our line of sight is too small to account for this, as it has a colour excess $E(B-V)=0.02 \mathrm{mag}$ (Schlafly \& Finkbeiner 2011), reported by NASA/IPAC Extragalactic Database (NED). Therefore, the additional reddening in our line of sight is intrinsic to NGC 3227.

Before modelling the internal reddening in NGC 3227, we first fixed our model for the Milky Way reddening. We applied an ebv component in SPEX to model this reddening, which incorporates the extinction curve of Cardelli et al. (1989), including the update for near-UV given by O'Donnell (1994). The $E(B-V)$ was set to 0.02 and the scalar specifying the ratio of total to selective extinction $R_{V}=A_{V} / E(B-V)$ was fixed to 3.1.

To model the internal reddening in NGC 3227, we considered different extinction laws, which are shown in Fig. 4. They are: (1) the Milky Way extinction law (i.e. the ebv model); (2) the extinction curve of Crenshaw et al. (2001) derived for NGC 3227; (3) the Small Magellanic Cloud (SMC) extinction curve of Gordon et al. (2003) (the 'SMC Bar Average' version) 


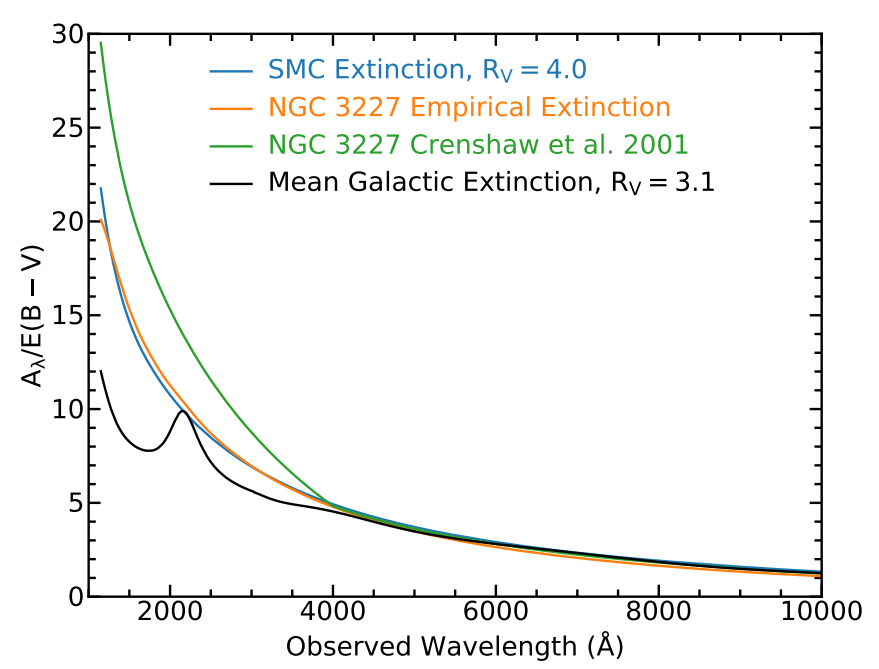

Fig. 4. Comparison of di erent dust extinction laws which were considered in our modelling of the optical-UV spectrum of NGC 3227. For modelling the internal reddening in the AGN with $E\left(\begin{array}{ll}B & V\end{array}\right)=045$, we use the SMC extinction law of Gordon et al. (2003) with $R_{V}=$ 40 (shown in blue). For foreground Milky Way extinction with $E(B V)=002$, we use the extinction curve of Cardelli et al. (1989), including the update for near-UV given by O Donnell (1994) (shown in black).

with $R_{V}=40$; (4) an empirical extinction curve that we determined from our HST COS and STIS spectra, assuming that the intrinsic spectrum is a power law with $f \quad 73$, which corresponds to the Jeans tail of a Shakura-Sunyaev disk spectrum.

Crenshaw et al. (2001) derived their extinction curve for NGC 3227 by comparison to NGC 4151, which they assumed had no internal extinction. As shown in Fig. 4, their curve and our empirical curve are both signi cantly steeper than the mean Milky Way extinction law and neither include a $2175 \AA$ bump . Therefore, the Galactic extinction curve is not suitable for correcting the internal reddening of NGC 3227. However, our empirically determined extinction curve is not as steep as that of Crenshaw et al. (2001). Instead, it closely matches an SMC extinction curve with $R_{V}=40$ (Fig. 4). Therefore, in our SED modelling we adopted this SMC extinction curve to correct for internal reddening in NGC 3227 . We note that internal extinction in AGN appears to be best described in general by an SMC extinction curve (Hopkins et al. 2004).

The user-de ned multiplicative model in SPEX (musr) was used to import the SMC extinction model into SPEX. From dbb continuum modelling of the HST COS and STIS spectra, as well as the OM data, we nd $E(B \quad V)=045003$ best ts the shape of the observed NIR-optical-UV data. The best- $t$ parameters of the $\mathrm{dbb}$ model are provided in Table 2 . In our tting of the continuum, we exclude regions in spectra where emission and absorption lines are present. In our modelling we included a template model component for the starlight emission from the bulge of the host galaxy, taken from Kinney et al. (1996), and allowed the normalisation of this component to be tted. As the OM data in the optical band are strongly dominated by the host galaxy starlight emission due to the larger aperture size than HST (see Fig. 3), we only use the STIS spectrum for modelling the continuum in the optical band. We nd STIS takes in a negligible amount of the starlight emission (Fig. 3), and thus it better facilitates modelling of the underlying optical continuum than

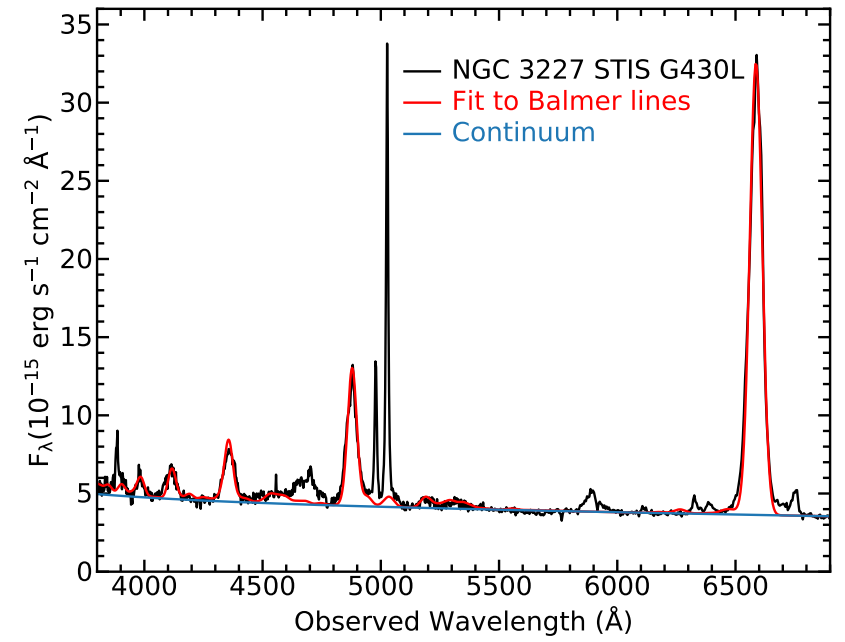

Fig. 5. Close-up of the STIS spectrum of NGC 3227 displayed in the observed frame, where the Balmer lines intensities have been tted using a Cloudy BLR model, reddened with an SMC extinction law with $R_{V}=40$ and best- $\mathrm{t}$ reddening of $E(B \quad V)=044$.

OM. In our SED modelling we included components to take into account contributions from $\mathrm{Fe}$ II and Balmer continuum emission in NGC 3227 (see Fig. 3). We refer to Mehdipour et al. (2015) where these models are described and applied to NGC 5548.

The internal reddening $E(B \quad V)=045$ derived from modelling the continuum is fully consistent with the reddening derived from the intensity of the Balmer lines in NGC 3227 (Fig. 5). Several studies have shown that the Balmer line ratios in the BLR of AGN are intrinsically unreddened and have an intensity ratio for $\mathrm{H} / \mathrm{H}$ comparable to the Case $\mathrm{B}$ value of 2.78 (Baker \& Menzel 1938) for a temperature of $10^{4} \mathrm{~K}$ (Dong et al. 2008; Gaskell 2017). The mean unreddened value is 2.72 with an observational dispersion of 03 (Gaskell 2017). To $t$ the full range of Balmer lines in the NGC 3227 spectrum, we use a typical BLR model calculated with Cloudy v17.00 (Ferland et al. 2017), using the unobscured spectral energy distribution of NGC 5548 (see Steenbrugge et al. 2005) with an ionisation parameter $U$ (Davidson 1977) of $\log U=108$, density $n_{\mathrm{H}}=10^{10} \mathrm{~cm}^{3}$, and a total column density of $N_{\mathrm{H}}=120 \quad 10^{22} \mathrm{~cm}^{2}$. This model gives an intrinsic (unreddened) $\mathrm{H} / \mathrm{H}$ intensity ratio of 2.58. In Fig. 5 the tted components are a power-law continuum, Fe II emission, and $E(B \quad V)$ for an SMC extinction law with $R_{V}=40$. The best $\mathrm{t}$ we obtain for the line ratios is $E(B \quad V)=044$. Allowing for the observed scatter in AGN Balmer line ratios, our tted extinction has an uncertainty of 0.05 . Our best- $t$ value is remarkably almost the same as the value obtained from modelling the shape of the full observed optical-UV continuum described earlier. So the derived $E\left(\begin{array}{ll}B \quad V\end{array}\right)$ based on the lines alone in the optical region are consistent with the same extinction law used to model the whole continuum spectrum from NIR to far-UV. In Sect. 4.1 we will further discuss the nature and origin of internal reddening in NGC 3227.

Interestingly, apart from the reddened $\mathrm{dbb}$ component, we also nd evidence of an unreddened component. This component is seen as an excess emission above the dbb model towards the shortest wavelengths in the COS band (see Fig. 6). As shown in this gure by including an unreddened component at the far-UV we obtain a signi cantly better $t$ to the NIR-optical-UV spectrum. As the unreddened component is weak and only observable 


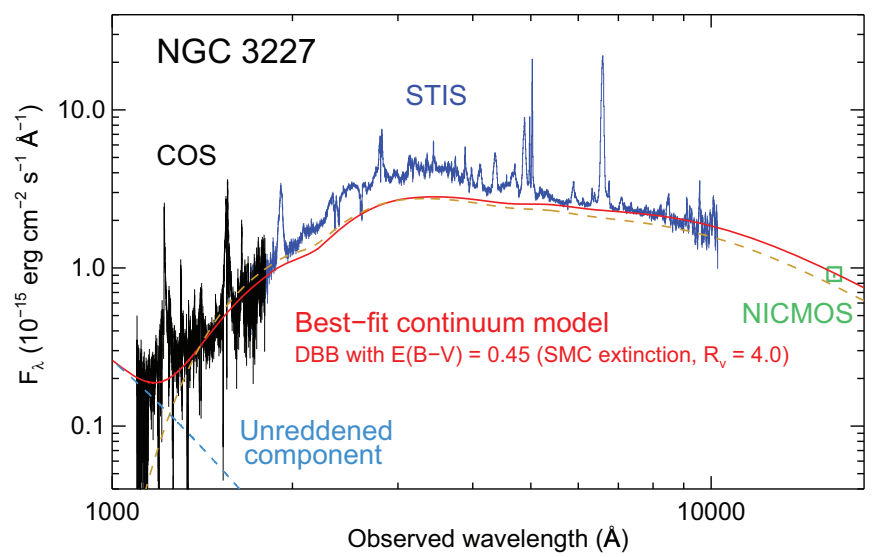

Fig. 6. NIR-optical-UV spectrum of NGC 3227 taken with HST COS and STIS. The continuum flux in the NIR from HST/NICMOS is also plotted. The best-fit continuum model, consisting of a reddened $\mathrm{dbb}$ and an additional unreddened component at shortest wavelengths (dashed blue line) is shown as a solid red line. For comparison the best-fit model without an unreddened component is also plotted (dashed brown line), which does not fit the data well at shortest wavelengths. The broad feature above the continuum at about $2000-4000 \AA$ is the blended Fe II and Balmer emission.

over a limited energy band in the far-UV, distinguishing between different possible models for this emission would be challenging. Over $1067-1300 \AA$, where this component is detected in the COS spectrum, its flux is about $3.4 \times 10^{-14} \mathrm{erg} \mathrm{cm}^{-2} \mathrm{~s}^{-1}$. This is about a factor of three larger than the reddened dbb flux. However, its flux is about three orders of magnitude smaller than the intrinsic dbb flux. As the flux of this unreddened component increases towards higher energies, it is likely it gets stronger in the extreme ultraviolet (EUV) band. To test this and estimate a maximum flux contribution for this component, we applied an unreddened version of the warm Comptonisation model that is used to model the EUV continuum and the 'soft X-ray excess' (see the comt modelling described in Sect. 3.2). The inclusion of this unreddened component improves the fit by $\Delta \chi^{2}=1300$. We find such an unreddened comt model, which fits the excess emission in the COS band well, has a flux that is still about two orders of magnitude smaller than the intrinsic SED model in the EUV band. Therefore, this unreddened component, which likely originates far from the central ionising source, is much weaker than the primary SED continuum and thus for the purpose of photoionisation it is effectively negligible. In Sect. 4.1 we will further discuss the possible origin and location of this unreddened component that is seen in the COS spectrum of NGC 3227.

In order to examine how our dbb continuum model extends to lower NIR energies beyond the STIS band, we made use of continuum fluxes that were derived by Kishimoto et al. (2007) using archival HST Near Infrared Camera and Multi-Object Spectrometer (NICMOS) observations, taken with the F160W

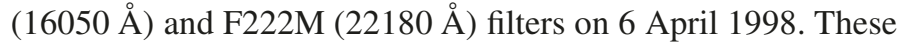
data are not included in our spectral fitting, but rather are overplotted for comparison with our SED model. As shown in Figs. 6 and 7 (right middle panel), the measured continuum at $16050 \AA$ is consistent with our dbb model. However, at the longer wavelength of $22180 \AA$, the flux of the measured continuum is higher than that of the dbb model (see Fig. 7, right middle panel). This excess above the dbb model is likely the high-energy tail of a thermal emission component from the hottest regions of the
Table 2. Best-fit parameters of the broadband continuum model components for NGC 3227, derived from modelling the 2016 and 2019 observations.

\begin{tabular}{|c|c|}
\hline Parameter & Value \\
\hline \multicolumn{2}{|c|}{ Disk blackbody component for NIR-optical-UV continuum (dbb): } \\
\hline \multirow[t]{2}{*}{ Normalisation $A$} & 7.3 (2016, fixed) \\
\hline & $4.0 \pm 0.2(2019)$ \\
\hline \multirow[t]{2}{*}{$T_{\max }(\mathrm{eV})$} & $10.2(2016$, coupled $)$ \\
\hline & $10.2 \pm 0.2(2019)$ \\
\hline \multicolumn{2}{|c|}{ Warm Comptonisation component for 'soft X-ray excess' (comt): } \\
\hline \multirow[t]{2}{*}{ Normalisation } & $5.9 \pm 0.1(2016)$ \\
\hline & $1.3 \pm 0.1(2019)$ \\
\hline$T_{\text {seed }}(\mathrm{eV})$ & 10.2 (coupled) \\
\hline \multirow[t]{2}{*}{$T_{\mathrm{e}}(\mathrm{keV})$} & $0.10 \pm 0.01(2016)$ \\
\hline & $0.10(2019$, coupled $)$ \\
\hline Optical depth $\tau$ & 30 (fixed) \\
\hline \multicolumn{2}{|c|}{ Primary X-ray power-law component (pow): } \\
\hline \multirow[t]{2}{*}{ Normalisation } & $38.7 \pm 0.1(2016)$ \\
\hline & $6.4 \pm 0.1(2019)$ \\
\hline \multirow[t]{2}{*}{ Photon index $\Gamma$} & $1.83 \pm 0.02(2016)$ \\
\hline & $1.70 \pm 0.03(2019)$ \\
\hline \multicolumn{2}{|c|}{$\mathrm{X}$-ray reflection component (refl): } \\
\hline \multirow{4}{*}{$\begin{array}{l}\text { Incident power-law Norm. } \\
\text { Incident power-law } \Gamma \\
\text { Reflection scale } s\end{array}$} & 38.7 (coupled) \\
\hline & 1.83 (coupled) \\
\hline & $0.61 \pm 0.02(2016)$ \\
\hline & $0.25 \pm 0.02(2019)$ \\
\hline \multicolumn{2}{|c|}{ C-stat $/$ expected C-stat $=4085 / 3272(2016)$} \\
\hline \multicolumn{2}{|c|}{ C-stat $/$ expected C-stat $=3891 / 3487(2019)$} \\
\hline
\end{tabular}

Notes. The disk blackbody dbb normalisation is in $10^{26} \mathrm{~cm}^{2}$. The normalisation of the $2016 \mathrm{dbb}$ component is fixed at a factor of 1.82 larger than that of the $2019 \mathrm{dbb}$ component based on the observed OM UVW1 fluxes. The power-law normalisation of the pow and refl components is in units of $10^{49}$ photons s $\mathrm{s}^{-1} \mathrm{keV}^{-1}$ at $1 \mathrm{keV}$. The normalisation of the Comptonisation component (comt) is in units of $10^{53}$ photons $\mathrm{s}^{-1} \mathrm{keV}^{-1}$. The high-energy exponential cut-off of the power-law for both pow and refl is fixed to $309 \mathrm{keV}$. The seed photon temperature $T_{\text {seed }}$ of comt is coupled to the maximum temperature $T_{\max }$ of $\mathrm{dbb}$. The normalisation of the incident power-law for the reflection component (refl) is coupled to that of the observed primary power-law continuum (pow). The intrinsic bolometric luminosity of each continuum component is as follows. For the 2016 observation: dbb: $3.4 \times 10^{43} \mathrm{erg} \mathrm{s}^{-1}$; comt: $5.1 \times 10^{42} \mathrm{erg} \mathrm{s}^{-1}$; pow: $7.1 \times 10^{42} \mathrm{erg} \mathrm{s}^{-1}$; refl: $1.3 \times 10^{42} \mathrm{erg} \mathrm{s}^{-1}$. For the $2019 \mathrm{ob}-$ servation: dbb: $1.9 \times 10^{43} \mathrm{erg} \mathrm{s}^{-1}$; comt: $1.1 \times 10^{42} \mathrm{erg} \mathrm{s}^{-1}$; pow: $1.6 \times 10^{42} \mathrm{erg} \mathrm{s}^{-1}$; refl: $5.4 \times 10^{41} \mathrm{erg} \mathrm{s}^{-1}$.

AGN torus. Indeed by going to longer wavelengths the SED becomes dominated by emission from the torus rather than the accretion disk, as shown for example in Mehdipour \& Costantini (2018) for IC 4329A. Since in the current paper we are interested in the primary emission from the accretion disk and the associated X-ray emission, we do not extend our spectral coverage to lower energies in the mid-IR to model the torus emission.

\subsection{X-ray continuum and absorption}

We simultaneously model XMM-Newton EPIC-pn, RGS, and $N U S T A R$ spectra of each observation. We took into account the 

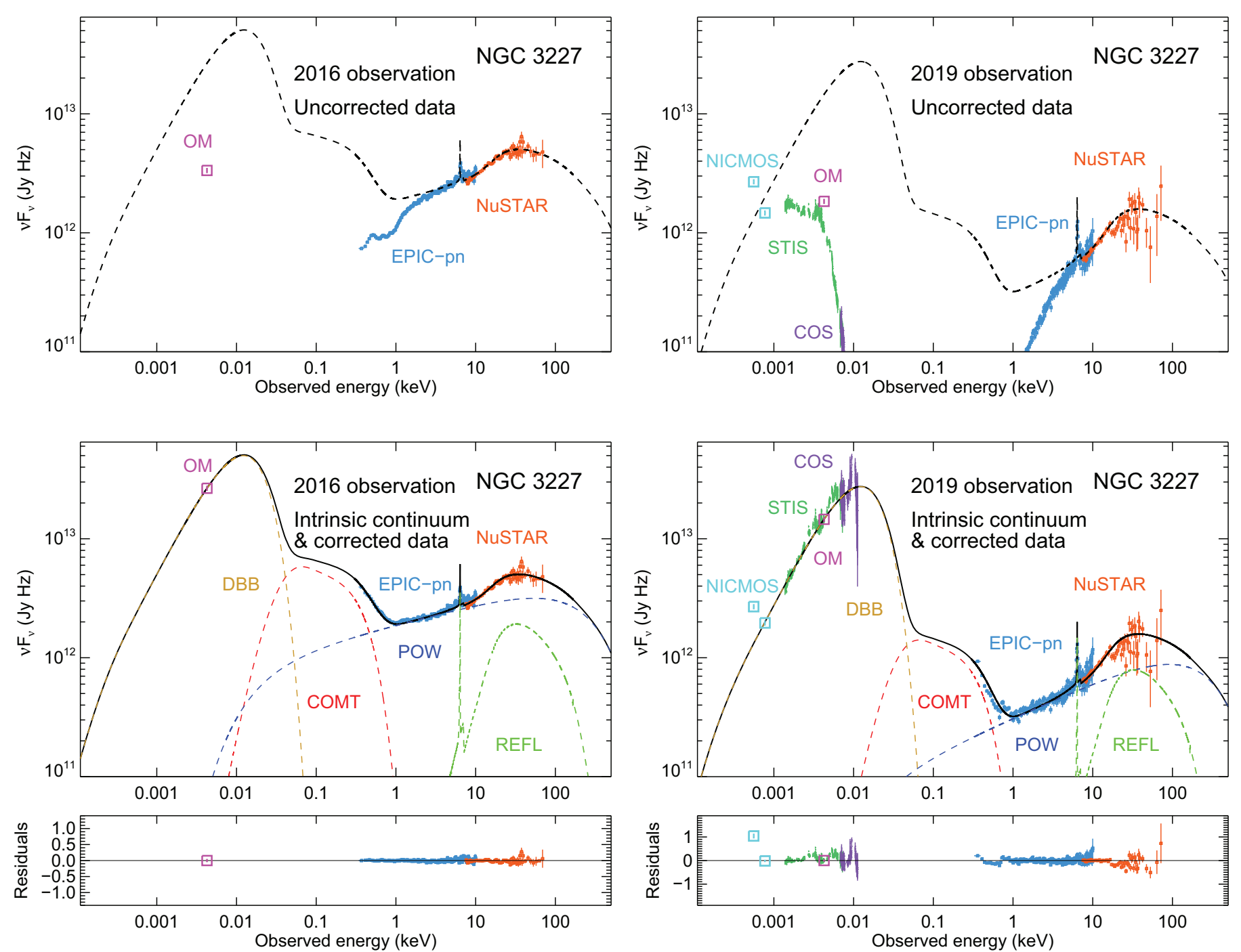

Fig. 7. SED continuum model of NGC 3227 from NIR to hard X-rays, derived from fitting the XMM-Newton, NuSTAR and HST data. The data are from the 2016 unobscured (left panels) and 2019 obscured (right panels) observations. The data in the top panels include the effects of reddening and X-ray absorption, while those in the middle panels are corrected for these effects, revealing the underlying continuum. The best-fit continuum model is shown in dashed black line in the top panels and solid black line the middle panels for comparison with the data. Residuals of the fit, defined as (data-model)/model, are displayed in the bottom panels. The archival HST NICMOS data at NIR are not included in our spectral fitting, but rather are over-plotted for comparison with our SED model as described in Sect. 3.1. The displayed spectra have been binned for clarity of presentation. The displayed OM data are taken with the UVW1 filter. The contribution of individual continuum components is displayed in the middle panels: a disk blackbody component (dbb, dashed brown line), a warm Comptonisation component for the 'soft X-ray excess' (comt, dashed red line), a power-law continuum for the hard X-rays (pow, dashed blue line), an X-ray reflection component (refl, dashed green line).

instrumental flux calibration differences by comparing the flux level of the instruments at their overlapping energy bands and re-scaling their cross-normalisation. The NuSTAR/EPIC-pn flux ratio was 1.09 in 2016 and 1.11 in 2019; the RGS/EPIC-pn flux ratio was 0.96 in 2016 and 0.91 in 2019 . To fit the X-ray spectra of NGC 3227 we started with a power-law continuum (pow). The $\mathrm{X}$-ray power-law model represents Compton up-scattering of the disk photons in an optically-thin, hot, corona. The high-energy exponential cut-off of the power-law was first set to $309 \mathrm{keV}$ (Turner et al. 2018), which is consistent with the NuSTAR data. We then tested freeing this parameter in our modelling, however, since it did not significantly improve the fit we kept it frozen at $309 \mathrm{keV}$ to limit the number of free parameters. A low-energy exponential cut-off was also applied to the power-law continuum to prevent it exceeding the maximum energy of the seed dbb disk photons $(10 \mathrm{eV})$. The photon index $\Gamma$ of the intrinsic power-law is $1.83 \pm 0.02$ in 2016 and $1.70 \pm 0.03$ in 2019 .

In addition to the power-law, the soft X-ray continuum of NGC 3227 shows the presence of a 'soft X-ray excess' component (see e.g. Markowitz et al. 2009; Noda et al. 2014). To model the soft excess in NGC 3227, we used a warm Comptonisation model (comt in SPEX), which has been used to model the soft excess in similar Seyfert-1 AGN, such as Mrk 509 (Mehdipour et al. 2011); NGC 5548 (Mehdipour et al. 2015); NGC 3783 (Mehdipour et al. 2017). In this explanation of the soft excess, the seed disk photons are up-scattered in a warm, optically thick, corona to produce the EUV continuum and the soft X-ray excess as its high-energy tail. Multi-wavelength studies have found warm Comptonisation to be a viable explanation for the soft Xray excess in Seyfert-1 AGN (e.g. Ark 120, Porquet et al. 2018). 
For more details about warm Comptonisation modelling of the soft X-ray excess, see e.g. Magdziarz et al. (1998); Mehdipour et al. (2011); Done et al. (2012); Petrucci et al. (2013, 2018, 2020); Kubota \& Done (2018). We note that this is only one plausible explanation proposed in the literature for the origin of the soft X-ray excess in AGN (relativistic ionised re ection is another explanation), yet it is su cient for our purpose of deriving a broadband SED for NGC 3227 in this paper. The parameters of the comt model are its normalisation, seed photon temperature $T_{\text {seed }}$, electron temperature $T_{\mathrm{e}}$, and optical depth of the upscattering plasma. In our modelling the $T_{\text {seed }}$ parameter of comt is coupled to the $T_{\max }$ parameter of the dbb model that ts the NIR-optical-UV data (Sect. 3.1). Because in the 2019 observation the soft X-ray spectrum is obscured, it can be challenging to constrain the parameters of the soft X-ray excess. Thus, we couple the 2019 comt plasma parameters to those of the unobscured 2016 observation and only allow its normalisation to be tted. Furthermore, as there can be degeneracy between plasma parameters of the soft excess model, we x optical depth of the optically-thick plasma to a ducial value of 30 , in order to limit the number of free parameters while still providing a good $t$. The best- t parameters of the power-law component (pow), the warm Comptonisation component (comt), and the disk blackbody model (dbb, described in Sect. 3.1) are provided in Table 2. The continuum components of our SED model, from NIR to hard X-rays, are displayed in Fig. 7 (middle panels).

The primary X-ray continuum undergoes reprocessing, which is evident by the presence of the Fe $\mathrm{K}$ line and the Compton hump in the XMM-Newton and NuSTAR spectra (Fig. 7). We applied an X-ray re ection component (refl in SPEX), which reprocesses an incident power-law continuum to $t$ the $\mathrm{Fe} \mathrm{K}$ line and the Compton hump at hard X-rays. The refl model computes the Fe K line according to Zycki \& Czerny (1994), and the Compton-re ected continuum according to Magdziarz \& Zdziarski (1995), as described in Zycki et al. (1999). The exponential high-energy cut-o of the incident power-law was also set to that of the observed primary power-law component at $309 \mathrm{keV}$ (Turner et al. 2018). In our modelling the normalisation of the incident power-law continuum was coupled to that of the 2016 observed power-law. The ionisation parameter of refl is set to zero to produce a cold re ection component with all abundances kept at their solar values. The photon index of the incident power-law is xed to that of the primary power-law seen in the unobscured 2016 observation. The re ection scale factor $(s)$ of the refl model was tted for the 2016 and 2019 observations. The best- $t$ parameters are provided in Table 2 .

In our spectral modelling we take into account the X-ray continuum and line absorption by the di use interstellar medium (ISM) in the Milky Way. This is done using the hot model in SPEX (de Plaa et al. 2004; Steenbrugge et al. 2005). This model calculates the transmission of a plasma in collisional ionisation equilibrium at a given temperature, which for neutral ISM is set to the minimum temperature of the model at $0.008 \mathrm{eV}$. The Galactic $N_{\mathrm{H}}$ in our line of sight towards NGC 3227 was xed to $20710^{20} \mathrm{~cm}^{2}$ (Murphy et al. 1996).

Absorption by ionised AGN out ows is evident in NGC 3227 (see. e.g. Turner et al. 2018). In our SED modelling we take into account absorption by the persistent warm-absorber out ow, and the additional absorption by the transient obscuring wind in the new 2019 data. The X-ray spectra of NGC 3227 do not show evidence of any detectable absorption by neutral gas in the ISM of the host galaxy. As noted in Sect. 1, the study of the ionised out ows are presented in detail separately in Paper II (Wang et al. 2021) for the warm absorber, and Paper III (Mao et al. in prep) for the new obscuring wind. Below we brie $y$ describe how the X-ray absorption by these ionised out ows were taken into account in our SED modelling.

For photoionisation and spectral modelling, we use the pion model in SPEX (see Mehdipour et al. 2016), which is a selfconsistent model that calculates both the photoionisation solution and the spectrum of a plasma in photoionisation equilibrium (PIE). The pion model uses the SED continuum model that is tted to the data (Fig. 7, middle panels), thus both the continuum and the absorption spectrum are tted together. The warm-absorber model that we use is based on the model derived from archival XMM-Newton/RGS observations of NGC 3227 (Paper II, Wang et al. 2021). This warm-absorber model consists of four di erent ionisation components (i.e. four pion components). There are no signi cant soft X-ray emission lines detected in the RGS spectrum of NGC 3227.

The column density $N_{\mathrm{H}}$ and the ionisation parameter (Krolik et al. 1981) of the warm-absorber components of the Wang et al. (2021) model were freed in our modelling of the 2016 unobscured observation to improve the $\mathrm{t}$ to our data. The total $N_{\mathrm{H}}$ of the warm absorber is found to be about $4810^{21} \mathrm{~cm}^{2}$, distributed over four ionisation components:
(A) $\log =30 \quad 01$ and $N_{\mathrm{H}}=10 \quad 01 \quad 10^{21} \mathrm{~cm}^{2}$;
(B) $\log =27 \quad 01$ and $N_{\mathrm{H}}=12 \quad 02 \quad 10^{21} \mathrm{~cm}^{2}$;
(C) $\log =18 \quad 01$ and $N_{\mathrm{H}}=97 \quad 09 \quad 10^{20} \mathrm{~cm}^{2}$;

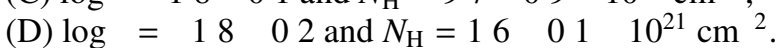

To model the new obscuration in the 2019 data one additional pion component is required, which is partially covering the $\mathrm{X}$-ray source. This is needed to be able to $\mathrm{t}$ the characteristic curvature seen in the 2019 broadband X-ray spectrum (Figs. 2 and 7). In our modelling of the 2019 obscured observation, the ionisation parameter of the above warm-absorber components were self-consistently lowered in response to the shielding of the ionising SED by the obscurer. Thus, for the 2019 observation the warm-absorber parameters are not tted, but rather the ionisation parameter is automatically re-calculated by the pion model for the obscured SED. The new ionisation parameter of the above components corresponds to $\log$ of $2.4,2.1$, 1.2 , and 24 , respectively. So the 2016 warm-absorber model is de-ionised in the 2019 observation, thus producing stronger absorption than in 2016. Such de-ionisation of the warm absorber by the obscurer has been previously presented for the obscuration in NGC 5548 (Kaastra et al. 2014) and NGC 3783 (Mehdipour et al. 2017). Our tted pion parameters of the obscurer in NGC 3227 are: $N_{\mathrm{H}}=54 \quad 10^{22} \mathrm{~cm}^{2}, \log =10$, and covering fraction $C_{f}=064$. More details about the new obscuring wind and its variability in NGC 3227 will be presented in our follow-up Paper III (Mao et al. in prep) and Paper IV (GraftonWaters et al. in prep).

Finally, we tested how much our SED model changes if an ionised relativistically-blurred re ection component is added to our model, where it would contribute to the soft X-ray excess (see e.g. Patrick et al. 2012; Beuchert et al. 2015). For this test we added a second refl component and allowed its ionisation parameter to be tted with relativistic disk broadening. We found the change on the photon index of the power-law and ux of the SED in the soft and hard X-ray bands are at levels of 1 to $3 \%$. This amount of change, which is comparable to the uncertainty of the continuum parameters, is not signi cant for photoionisation modelling (see e.g. Kara et al. 2021). Therefore, considering the above test, and lack of a consensus in previous publications of NGC 3227 about the nature of the soft X-ray excess, we keep our SED model as it was before the test. Our aim in this paper is not distinguishing between di erent models for the soft excess 
in NGC 3227, but rather adopt one feasible model for producing the overall SED.

\section{Discussion}

\subsection{Nature and origin of dust extinction in NGC 3227}

We showed in Sect. 3.1 that the optical-UV spectra of NGC 3227 display strong internal reddening. This is evident in both the shape of the continuum (Figs. 6 and 7) and the Balmer lines (Fig. 5). Here we discuss the possible origin and location of this dust reddening in NGC 3227. The host galaxy of NGC 3227 is interacting with its companion galaxy NGC 3226. The H I radio mapping studies of NGC 3227 show interesting tidal interactions between the two galaxies. As a result there is a signi cant column density of neutral gas in the host galaxy of NGC 3227. According to radio observations, the $\mathrm{H}_{\mathrm{I}} 21-\mathrm{cm}$ absorption suggests a neutral gas column density of $55 \quad 10^{21} \mathrm{~cm}^{2}$ along our line of sight (Mundell et al. 1995b). However, interestingly, our line of sight to the X-ray and UV source does not show the presence of any signi cant amount of intrinsic neutral gas as discussed below. Apart from absorption by ionised AGN out ows (Sect. 3.2), the X-ray spectra of NGC 3227 do not show evidence of any additional absorption by neutral gas, such as in the ISM of NGC 3227. This is also supported from HST UV spectroscopy of the intrinsic Ly line in NGC 3227, which shows strong absorption, but does not have broad damping wings. This implies a total intrinsic H I column of $<10^{19} \mathrm{~cm}^{2}$. The most feasible explanation for this discrepancy between the $\mathrm{H}_{\mathrm{I}}$ absorption in radio, and lack of $\mathrm{H}_{\mathrm{I}}$ absorption in X-rays and $\mathrm{UV}$, is that the radio continuum emission is not coincident with the nucleus of NGC 3227, where the X-ray and UV point source reside. Indeed the high resolution radio observations of Mundell et al. (1995a) show that the radio emission is extended along the direction of the ionization cone de ned by [O III] 5007 and the extended X-ray emission (Zeng et al. 2003).

Since the internal reddening $E(B \quad V)=045$ cannot be associated with neutral ISM gas in NGC 3227, one likely explanation is that the internal reddening is linked to nuclear gas, likely the out owing warm-absorber gas that may originate from the dusty torus of NGC 3227 . The relation between the hydrogen column density $N_{\mathrm{H}}$ and reddening $E(B \quad V)$ can be written as $N_{\mathrm{H}}\left(\mathrm{cm}^{2}\right)=a \quad 10^{21} E(B \quad V)(\mathrm{mag})$, where $a$ is reported in the literature to have a value ranging from about 5.5 to 6.9 (Bohlin et al. 1978; Gorenstein 1975; Predehl \& Schmitt 1995; Güver \& Özel 2009). Therefore, for our derived $E(B \quad V)=045$, the expected $N_{\mathrm{H}}$ is about $3 \quad 10^{21} \mathrm{~cm}^{2}$. Interestingly, this $N_{\mathrm{H}}$ matches the column density $N_{\mathrm{H}}$ of the lowerionisation components of the warm absorber in NGC 3227 (see Sect. 3.2, and also Turner et al. 2018 and our Paper II by Wang et al. 2021). Such internal reddening without neutral Xray absorption was also seen in Seyfert-1 galaxies MCG -6-3015 (Lee et al. 2001), ESO 113-G010 (Mehdipour et al. 2012), and IC 4329A (Mehdipour \& Costantini 2018). Similarly, in these objects the internal reddening was explained by the lowerionisation components of their warm-absorber out ows being dusty. In contrast to Crenshaw et al. (2001), who attribute the extinction and absorption to interstellar gas far from the nucleus in NGC 3227, we deduce that the internal reddening in NGC 3227 can be attributed to the presence of dust in the warmabsorber out ows, likely being driven from the AGN torus. This dusty out ow scenario is further supported by the recent study of Alonso-Herrero et al. (2019), and references therein, which show in NGC 3227 there are nuclear molecular out ows, as well as polar dust emission in the mid-IR, likely coinciding with the AGN ionisation cone.

We noted in Sect. 3.1 that the HST/COS spectrum of NGC 3227 contains an unreddened component in the far-UV (Fig. 6). An alternative explanation to this unreddened component may be a custom dust extinction law, one that is di erent from the ones considered in Fig. 4, including the SMC extinction with $R_{V}=40$ that we used for de-reddening the spectra. Such a custom extinction curve would describe the intrinsic spectrum as a power law in $F$ with index $=73$ to match the red tail of the unreddened component. With such a custom extinction law the presence of the unreddened scattered continuum component is not essential. However, there are two points that favour the scattered radiation interpretation. Firstly, this component is needed to properly $t$ the optical/UV continuum when using a standard extinction law (Fig. 6). Secondly, the extended X-ray emission seen in the Chandra image of NGC 3227 (Zeng et al. 2003), and the extended NLR emission, imply the existence of a cone of scattered light similar to other nearby obscured Seyferts, such as NGC 4151 and NGC 1068. The study of Alonso-Herrero et al. (2019) shows the presence of this cone in NGC 3227. Therefore, we deduce that the unreddened component detected in the HST COS spectrum is a real scattered emission component, likely originating from the extended NLR of NGC 3227.

\subsection{Broadband continuum emission of NGC 3227}

The broadband continuum of NGC 3227 is strongly modi ed by internal reddening and X-ray absorption. By modelling these effects in this paper, the underlying intrinsic emission of the AGN is uncovered. The NIR-optical-UV continuum of NGC 3227, measured thanks to broad HST spectral coverage, is fully consistent with a disk blackbody $(\mathrm{dbb})$ model. In order to $\mathrm{t}$ the soft $\mathrm{X}$ ray excess, an additional spectral component is required, which in this paper we modelled with a warm Comptonisation component (comt). Our modelling of the XMM-Newton and $\mathrm{NuS}$ $T A R$ spectra shows that the hard X-ray spectrum of NGC 3227 is consistent with a typical AGN X-ray power-law, produced in an optically-thin, hot, corona. The X-ray power-law is accompanied by a neutral, narrow, X-ray re ection component, producing the observed $\mathrm{Fe} \mathrm{K}$ line and the Compton hump at higher energies.

We note that the broadband continuum model presented in this paper should be considered as only one feasible explanation. The primary purpose of Paper I is to establish the SED that is needed for the photoionization modelling of the out ows in NGC 3227, which are studied in more detail in our subsequent papers. To this end the single SED model presented in this paper is su cient for the purpose of our investigation. Furthermore, considering the strong reddening in NGC 3227, and the caveats in modelling it, as well complexities in the strong X-ray absorption, NGC 3227 is not the best candidate for evaluating merits of di erent models for the soft X-ray excess and thus tackling the long-standing issue in deciphering its origin in AGN.

From the $\mathrm{dbb}$ model we can derive the inner disk radius and the accretion rate using the tted parameters of the $\mathrm{dbb}$ model. The radial temperature pro le of the accretion disk is given by $T(r)=3 G M M\left[1 \quad\left(R_{\text {in }} r\right)^{12}\right]\left(8 r^{3}\right)^{14}$ where $T$ is the local temperature at radius $r$ in the disk, $G$ the gravitational constant, $M$ the mass of the $\mathrm{SMBH}, M$ the accretion rate, the Stefan-Boltzmann constant, and $R_{\text {in }}$ is the radius at the inner edge of the disk. The ux at frequency from the disk seen by an observer at distance $d$ is given by $F=\left[\begin{array}{llll}4 & h^{3} \cos i\left(c^{2} d^{2}\right)\end{array}\right] \int_{R_{\text {in }}}^{R_{\text {out }}}\left(\mathrm{e}^{h} k T(r) \quad 1\right)^{1} r \mathrm{~d} r$ where $i$ is the 
inclination angle of the disk, $R_{\text {out }}$ the radius at the outer edge of the disk, $h$ the Planck constant, and $c$ the speed of light. The parameter $R_{\text {out }}$ cannot be constrained by spectral tting since most of the radiation originates from the inner regions of the disk, thus $R_{\text {out }}$ is xed to $10^{3} R_{\text {in }}$. The tted parameters of the dbb model are then the normalisation $A=R_{\text {in }}^{2} \cos i$ and the maximum temperature of the disk $T_{\max }$, which occurs at $r=\left(\begin{array}{lll}49 & 36\end{array}\right) R_{\text {in }}$.

Therefore, using our best- $\mathrm{t}$ normalisation $A$ value for the 2019 observation (Table 2), for which $\mathrm{dbb}$ is better constrained thanks to HST spectral coverage, and the black hole mass of $M=59610^{6} M$ for NGC 3227 (Bentz \& Katz 2015), we nd $R_{\text {in }}=\left(\begin{array}{ll}113 & \overline{\cos i}\end{array}\right) R_{\mathrm{g}}$, where $R_{\mathrm{g}}=2 G M c^{2}$. Adopting the inclination angle of 30 from Alonso-Herrero et al. (2019) for the nuclear ionisation cone of NGC 3227, we get $R_{\text {in }}=12 R_{\mathrm{g}}$, with a propagated uncertainty of about $1 R_{\mathrm{g}}$. This suggests the disk in NGC 3227 is likely truncated. Using the above expression for $T(r)$, and our best- t $T_{\max }$ (Table 2) and $R_{\text {in }}$ value, the mass accretion rate $M$ can be obtained, which is about $0.1 M \mathrm{yr}^{1}$ according to our dbb model.

Our broadband continuum modelling of the 2019 observation gives an intrinsic bolometric luminosity of $2210^{43} \mathrm{erg} \mathrm{s}^{1}$. In the 2016 observation the intrinsic luminosity was higher by a factor of 2.1 than in the 2019 observation. The individual continuum components contribute to the bolometric luminosity in 2019 as following: $85 \%$ from the disk blackbody (dbb), 5\% from the warm Comptonisation component (comt), 7\% from the X-ray power-law (pow), and $3 \%$ from the re ection component (refl). Taking into account the black hole mass of $5.9610^{6} M$ (Bentz \& Katz 2015), the 2019 bolometric luminosity in NGC 3227 corresponds to about $3 \%$ of the Eddington luminosity. Our derived bolometric luminosity and the Eddington ratio matches the SED modelling results of Vasudevan et al. (2010): 2-3 $10^{43} \mathrm{erg} \mathrm{s}^{1}$ and 2-4\% Eddington luminosity.

\section{Conclusions}

In 2019 we found a transient obscuration event in Seyfert-1 galaxy NGC 3227 using our Swift monitoring programme of a sample of AGN. Follow-up ToO observations were triggered with XMM-Newton, NuSTAR, and HST. Here in Paper I we have determined the SED of NGC 3227, which is required for investigating the properties and origin of the new obscuring gas. We have modelled the broadband continuum of NGC 3227 using HST, XMM-Newton, and NuSTAR spectra extending from NIR $(10255 \AA)$ to hard X-rays $(78 \mathrm{keV})$. We make use of both archival unobscured (2016) and new obscured (2019) spectra to disentangle the spectral components of the SED. We took into account all reddening and absorption processes that take place in our line of sight towards the nucleus of NGC 3227. Based on the results of our investigation we conclude the following points.

1. The underlying NIR-optical-UV continuum of NGC 3227 is tted well with a disk blackbody model with a maximum temperature of about $10 \mathrm{eV}$, inner disk radius of $12 R_{\mathrm{g}}$, and an accretion rate of $0.1 M \mathrm{yr}^{1}$. The EUV continuum and the soft X-ray excess in NGC 3227 are consistent with a warm Comptonisation component, up-scattering the disk seed photons in a warm, optically-thick corona.

2. Our SED continuum modelling shows that the AGN radiation in NGC 3227 is internally reddened with $E\left(\begin{array}{ll}B & V\end{array}\right)=$ 045 . This reddening, which is seen in both the continuum and the Balmer lines, is most consistent with an SMC extinction law with $R_{V}=40$. We nd the internal reddening in
NGC 3227 most likely occurs in dusty ionised out ows from the AGN torus, rather than being caused by neutral gas in the ISM of the host galaxy.

3. Our modelling shows the presence of an unreddened farUV continuum component at the shortest wavelengths of the COS spectrum. This component is likely scattered radiation from the extended NLR of NGC 3227.

4. From our modelling of the intrinsic broadband continuum in NGC 3227, we derive a bolometric luminosity of about $2210^{43} \mathrm{erg} \mathrm{s}^{1}$ in 2019, which corresponds to about $3 \%$ of the Eddington luminosity.

Acknowledgements. This work is based on observations obtained with XMMNewton, an ESA science mission with instruments and contributions directly funded by ESA Member States and the USA (NASA). This research has made use of data obtained with the NUSTAR mission, a project led by the California Institute of Technology (Caltech), managed by the Jet Propulsion Laboratory (JPL) and funded by NASA. We thank the Swift team for monitoring our AGN sample, and the XMM-Newton, NuSTAR, and HST teams for scheduling our ToO triggered observations. This work was supported by NASA through a grant for HST program number 15673 from the Space Telescope Science Institute, which is operated by the Association of Universities for Research in Astronomy, Incorporated, under NASA contract NAS5-26555. SRON is supported nancially by NWO, the Netherlands Organization for Scienti c Research. J.M. acknowledges the support from STFC (UK) through the University of Strathclyde UK APAP network grant ST/R000743/1. S.B. acknowledges nancial support from ASI under grants ASI-INAF I/037/12/0 and n. 2017-14-H.O. B.D.M. acknowledges support from Ramón y Cajal Fellowship RYC2018-025950-I. S.G.W. acknowledges the support of a PhD studentship awarded by the UK Science \& Technology Facilities Council (STFC). P.O.P. acknowledges nancial support from the CNES French space agency and the PNHE high energy national program of CNRS. G.P. acknowledges funding from the European Research Council (ERC) under the European Union s Horizon 2020 research and innovation programme (grant agreement No 865637). D.J.W. acknowledges support from STFC in the form of an Ernest Rutherford Fellowship. We thank the anonymous referee for the constructive comments.

\section{References}

Alonso-Herrero, A., García-Burillo, S., Pereira-Santaella, M., et al. 2019, A\&A, 628, A65

Arav, N., Chamberlain, C., Kriss, G. A., et al. 2015, A\&A, 577, A37

Baker, J. G. \& Menzel, D. H. 1938, ApJ, 88, 52

Bentz, M. C. \& Katz, S. 2015, PASP, 127, 67

Beuchert, T., Markowitz, A. G., Krauß, F., et al. 2015, A\&A, 584, A82

Blustin, A. J., Page, M. J., Fuerst, S. V., Branduardi-Raymont, G., \& Ashton, C. E. 2005, A\&A, 431, 111

Bohlin, R. C., Savage, B. D., \& Drake, J. F. 1978, ApJ, 224, 132

Cardelli, J. A., Clayton, G. C., \& Mathis, J. S. 1989, ApJ, 345, 245

Crenshaw, D. M., Kraemer, S. B., Bruhweiler, F. C., \& Ruiz, J. R. 2001, ApJ, 555,633

Dashtamirova, D. \& Fischer, W. 2020, Cosmic Origins Spectrograph Instrument Handbook, Version 12.1, Tech. rep., STScI, Baltimore

Davidson, K. 1977, ApJ, 218, 20

de Plaa, J., Kaastra, J. S., Tamura, T., et al. 2004, A\&A, 423, 49

de Vaucouleurs, G., de Vaucouleurs, A., Corwin, Herold G., J., et al. 1991, Third Reference Catalogue of Bright Galaxies

Dehghanian, M., Ferland, G. J., Kriss, G. A., et al. 2019, ApJ, 877, 119

den Herder, J. W., Brinkman, A. C., Kahn, S. M., et al. 2001, A\&A, 365, L7

Done, C., Davis, S. W., Jin, C., Blaes, O., \& Ward, M. 2012, MNRAS, 420, 1848

Dong, X., Wang, T., Wang, J., et al. 2008, MNRAS, 383, 581

Ferland, G. J., Chatzikos, M., Guzmán, F., et al. 2017, Rev. Mexicana Astron. Astro s., 53, 385

Gaskell, C. M. 2017, MNRAS, 467, 226

Gehrels, N., Chincarini, G., Giommi, P., et al. 2004, ApJ, 611, 1005

Gordon, K. D., Clayton, G. C., Misselt, K. A., Landolt, A. U., \& Wol , M. J. 2003, ApJ, 594, 279

Gorenstein, P. 1975, ApJ, 198, 95

Grafton-Waters et al. in prep, A\&A, (Paper IV)

Green, J. C., Froning, C. S., Osterman, S., et al. 2012, ApJ, 744, 60

Güver, T. \& Özel, F. 2009, MNRAS, 400, 2050

Harrison, F. A., Craig, W. W., Christensen, F. E., et al. 2013, ApJ, 770, 103

Hopkins, P. F., Strauss, M. A., Hall, P. B., et al. 2004, AJ, 128, 1112

Jansen, F., Lumb, D., Altieri, B., et al. 2001, A\&A, 365, L1 
Kaastra, J. S. \& Bleeker, J. A. M. 2016, A\&A, 587, A151

Kaastra, J. S., Kriss, G. A., Cappi, M., et al. 2014, Science, 345, 64

Kaastra, J. S., Mewe, R., \& Nieuwenhuijzen, H. 1996, in UV and X-ray Spectroscopy of Astrophysical and Laboratory Plasmas, ed. K. Yamashita \& T. Watanabe, 411-414

Kaastra, J. S., Raassen, A. J. J., de Plaa, J., \& Gu, L. 2020, SPEX X-ray spectral tting package, Zenodo, 10.5281/zenodo.3939056

Kara, E., Mehdipour, M., Kriss, G. A., et al. 2021, ApJ, submitted, arXiv:2105.05840

Kinney, A. L., Calzetti, D., Bohlin, R. C., et al. 1996, ApJ, 467, 38

Kishimoto, M., Hönig, S. F., Beckert, T., \& Weigelt, G. 2007, A\&A, 476, 713

Kriss, G. A., Mehdipour, M., Kaastra, J. S., et al. 2019, A\&A, 621, A12

Krolik, J. H., McKee, C. F., \& Tarter, C. B. 1981, ApJ, 249, 422

Kubota, A. \& Done, C. 2018, MNRAS, 480, 1247

Laha, S., Guainazzi, M., Dewangan, G. C., Chakravorty, S., \& Kembhavi, A. K. 2014, MNRAS, 441, 2613

Lee, J. C., Ogle, P. M., Canizares, C. R., et al. 2001, ApJ, 554, L13

Lobban, A. P., Turner, T. J., Reeves, J. N., Braito, V., \& Miller, L. 2020, MNRAS, 494, 5056

Lodders, K., Palme, H., \& Gail, H.-P. 2009, Landolt Börnstein, 44

Magdziarz, P., Blaes, O. M., Zdziarski, A. A., Johnson, W. N., \& Smith, D. A. 1998, MNRAS, 301, 179

Magdziarz, P. \& Zdziarski, A. A. 1995, MNRAS, 273, 837

Mao et al. in prep, A\&A, (Paper III)

Markowitz, A., Reeves, J. N., George, I. M., et al. 2009, ApJ, 691, 922

Markowitz, A. G., Krumpe, M., \& Nikutta, R. 2014, MNRAS, 439, 1403

Mason, K. O., Breeveld, A., Much, R., et al. 2001, A\&A, 365, L36

Mehdipour, M., Branduardi-Raymont, G., Kaastra, J. S., et al. 2011, A\&A, 534, A39

Mehdipour, M., Branduardi-Raymont, G., \& Page, M. J. 2012, A\&A, 542, A30

Mehdipour, M. \& Costantini, E. 2018, A\&A, 619, A20

Mehdipour, M., Kaastra, J. S., \& Kallman, T. 2016, A\&A, 596, A65

Mehdipour, M., Kaastra, J. S., Kriss, G. A., et al. 2017, A\&A, 607, A28

Mehdipour, M., Kaastra, J. S., Kriss, G. A., et al. 2015, A\&A, 575, A22

Mundell, C. G., Holloway, A. J., Pedlar, A., et al. 1995a, MNRAS, 275, 67

Mundell, C. G., Pedlar, A., Axon, D. J., Meaburn, J., \& Unger, S. W. 1995b, MNRAS, 277, 641

Murphy, E. M., Lockman, F. J., Laor, A., \& Elvis, M. 1996, ApJS, 105, 369

Noda, H., Makishima, K., Yamada, S., et al. 2014, ApJ, 794, 2

O Donnell, J. E. 1994, ApJ, 422, 158

Patrick, A. R., Reeves, J. N., Porquet, D., et al. 2012, MNRAS, 426, 2522

Petrucci, P. O., Gronkiewicz, D., Rozanska, A., et al. 2020, A\&A, 634, A85

Petrucci, P.-O., Paltani, S., Malzac, J., et al. 2013, A\&A, 549, A73

Petrucci, P. O., Ursini, F., De Rosa, A., et al. 2018, A\&A, 611, A59

Porquet, D., Reeves, J. N., Matt, G., et al. 2018, A\&A, 609, A42

Predehl, P. \& Schmitt, J. H. M. M. 1995, A\&A, 293, 889

Schla y, E. F. \& Finkbeiner, D. P. 2011, ApJ, 737, 103

Shakura, N. I. \& Sunyaev, R. A. 1973, A\&A, 24, 337

Steenbrugge, K. C., Kaastra, J. S., Crenshaw, D. M., et al. 2005, A\&A, 434, 569

Strüder, L., Briel, U., Dennerl, K., et al. 2001, A\&A, 365, L18

Turner, T. J., Reeves, J. N., Braito, V., et al. 2018, MNRAS, 481, 2470

Vasudevan, R. V., Fabian, A. C., Gandhi, P., Winter, L. M., \& Mushotzky, R. F. 2010, MNRAS, 402, 1081

Wang, Y., Kaastra, J. S., Mehdipour, M., et al. 2021, A\&A, submitted (Paper II) Woodgate, B. E., Kimble, R. A., Bowers, C. W., et al. 1998, PASP, 110, 1183

Zeng, J., George, I., \& Kraemer, S. B. 2003, Extended emission around nearby Seyfert-1 galaxies, 4 Years of Chandra Observations: A Tribute to Riccardo Giacconi, Proceedings of the symposium held in Huntsville, Alabama, USA, September 16-18, 2003, https://wwwastro.msfc.nasa.gov/Ch4/

Zycki, P. T. \& Czerny, B. 1994, MNRAS, 266, 653

Zycki, P. T., Done, C., \& Smith, D. A. 1999, MNRAS, 305, 231 